Conclusions Although SES had favourable angiographic outcomes at 6 months as compared with PES and ZES, these angiographic benefits were not translated into better clinical outcomes in patients with diabetes up to 2 years. Table: Six-month Angiographic and 2-year clinical outcomes Variable, n (\%) SES Group ( $\mathrm{n}=102$ pts, 247 lesions) PES Group ( $\mathrm{n}=138$ pts, 414 lesions) ZES Group ( $\mathrm{n}=65 \mathrm{pts}$, 138 lesions) $\mathrm{p}$ value Stent diameter, $\mathrm{mm} 2.95 \pm 0.362 .86 \pm 0.38$ $3.03 \pm 0.44<0.001$ Stent length, $\mathrm{mm} 26.87 \pm 6.49 \quad 25.61 \pm 7.08$ $24.10 \pm 5.870 .008$ Binary restenosis 12 (7.5) 40 (15.9) 16 (19.8) 0.013 Restenosis percent, \% $19.45 \pm 17.86 \quad 28.51 \pm 21.93 \quad 32.44 \pm 25.71$ $<0.001$ Late loss, mm $0.45 \pm 0.490 .85 \pm 1.720 .73 \pm 0.690 .008$ Cardiac death $2(2.0) 1(0.7) 1(1.5) 0.696 \mathrm{Q}$-wave MI 0 (0) 0 (0) 0 (0) 1.000 Non-Q-wave MI 0 (0) 0 (0) 0 (0) 1.000 TLR 6 (5.9) 16 (11.6) 9 (13.8) 0.190 TVR 8 (7.8) 22 (15.9) 12 (18.5) 0.092 TVR-MACE 13 (12.7) 25 (18.1) 15 (23.1) 0.218 Stent thrombosis 0 (0) 0 (0) 3 (4.6) 0.004.

Table Six-month Angiographic and 2-year clinical outcomes

\begin{tabular}{llllr}
\hline & $\begin{array}{l}\text { SES Group } \\
\text { (n=102 pts, }\end{array}$ & $\begin{array}{l}\text { PES Group } \\
\text { (n=138 pts, }\end{array}$ & $\begin{array}{l}\text { ZES Group } \\
\text { (n=65 pts, }\end{array}$ & \\
Variable, n (\%) & $\mathbf{2 4 7 \text { lesions) }}$ & $\mathbf{4 1 4}$ lesions) & $\mathbf{1 3 8 \text { lesions) }}$ & P value \\
\hline Stent diameter, mm & $2.95 \pm 0.36$ & $2.86 \pm 0.38$ & $3.03 \pm 0.44$ & $<0.001$ \\
Stent length, mm & $26.87 \pm 6.49$ & $25.61 \pm 7.08$ & $24.10 \pm 5.87$ & 0.008 \\
Binary restenosis & $12(7.5)$ & $40(15.9)$ & $16(19.8)$ & 0.013 \\
Restenosis percent, \% & $19.45 \pm 17.86$ & $28.51 \pm 21.93$ & $32.44 \pm 25.71$ & $<0.001$ \\
Late loss, mm & $0.45 \pm 0.49$ & $0.85 \pm 1.72$ & $0.73 \pm 0.69$ & 0.008 \\
Cardiac death & $2(2.0)$ & $1(0.7)$ & $1(1.5)$ & 0.696 \\
Q-wave Ml & $0(0)$ & $0(0)$ & $0(0)$ & 1.000 \\
Non-0-wave MI & $0(0)$ & $0(0)$ & $0(0)$ & 1.000 \\
TLR & $6(5.9)$ & $16(11.6)$ & $9(13.8)$ & 0.190 \\
TVR & $8(7.8)$ & $22(15.9)$ & $12(18.5)$ & 0.092 \\
TVR-MACE & $13(12.7)$ & $25(18.1)$ & $15(23.1)$ & 0.218 \\
Stent thrombosis & $0(0)$ & $0(0)$ & $3(4.6)$ & 0.004 \\
\hline
\end{tabular}

\section{e0473 RAPID RE-ENDOTHELIALIZATION AND ANTI-INTIMAL HYPERPLASIA CORONARY STENT SYSTEM WITH A NOVEL BIOMACROMOLECULAR PROHEALING COATING}

\section{doi:10.1136/hrt.2010.208967.473}

Shen Li, Yang Wei, Zhong Wei, Tian Wenjie, Tang Jingliang, Wu Yizhe. Shanghai Institute of Cardiovascular Diseases and Cardiology Department, Zhongshan Hospital, Shanghai, China

Background Rapid healing of endothelium on the coronary stent is a crucial method to prevent late stent thrombosis, a rare but lifethreatening complication of drug eluting stents (DES). Here we developed a novel biomacromolecular coating for pro-healing DES and investigated their anti-proliferation as well as re- endothelialisation function.

Methods A dual function DES was designed in an asymmetric coating way: a layer-by-layer (LBL) self-assembly polymer of chitosan/heparin (CS/HEP) coated onto aluminal side of stents to accelerate re-endothelialisation and sirolimus onto vessel wall side to inhibit neointimal hyperplasia. Morphological, gene transcript (RTPCR), endothelial and antithrombotic marker expression analyses were used to evaluate the effects of CS/HEP coating on adhesion, proliferation and differentiation of $\mathrm{CD}_{13}{ }^{+}$endothelial progenitor cells (EPCs). Finally, the prohealing function as well as impact on coronary stenosis of this stent system were assessed in porcine model.

Results CS/HEP coating can significantly promote the adhesion, proliferation and differentiation of EPCs in vitro. CS/HEP upregulated expression of endothelial marker (ie, PECAM-1 and eNOS) and antithrombotic factor (ie, thrombomodulin). Interestingly, CS/HEP also promoted down-regulation of sirtuin-1, a gene related with endothelial cellular senescence. In porcine model, CS/HEP modified sirolimus eluting stent (CH-SES) showed rapid endothelialization superiority to bare metal stent (BMS) and SES, even in 1 week after stent implantation. Through electron microscopy analysis, the arteries treated with CH-SES were mostly fully endothelialized. As for effects on intimal hyperplasia, by angiography, intravascular ultrasound and histomorphometric analysis, there was no significant difference between CH-SES and SES in intimal thickness from 1 month to 3 and 6 months. Generally, SES took at least 3-4 weeks for the endothelial coverage of the stent struts, while the $\mathrm{CH}$-SES only took 1-2 weeks for endothelial repair and kept antiproliferation function as SES.

Conclusion This natural chitosan/heparin biomacromolecular selfassembly coating was safe and efficient in stent implanted porcine model. The preliminary results hinted possible molecular basis of $\mathrm{CS} / \mathrm{HEP}$ for rapid endothelial recovery. Meanwhile, coated with heparin, the CS -SES showed potent anti-coagulation function compared to traditional SES. To sum up, CS -SES may represent a promising self-rapid healing DES system to prevent in stent thrombosis as well as restenosis.

\section{e0474 EFFECT OF PLAQUE DISTRIBUTION TO BIOMECHANICAL}

doi:10.1136/hrt.2010.208967.474

Cheng Xunming, He Guoxiang, Tong Shifei, Liu Jianping. Department of Cardiology, Southwest Hospital, The Third Military Medical University \& Chongqing Institute of Interventional Cardiology, Chongqing, China

Introduction we presume that the plaque vulnerability of mildly lesions will be related to its intrinsic structural features and biomechanical characteristics. However, very little is known about their relationship between structural features and plaque vulnerability and about effect of biomechanical characteristics and plaque behaviour on vulnerable plaque. It is well known that coronary angiography (CAG) cannot accurately determine lesion morphology because it only shows the silhouette of the contrast materiel passing through the stenotic lesions. In recent years, intravascular ultrasound (IVUS) has evolved as a valuable adjunct to angiography. IVUS allows precise tomographic measurement of lumen area and plaque size, distribution and, to some extent, composition. Therefore, IVUS provide us likelihood for study on structural features and biomechanics characteristics in angiographic mildly stenosis in vivo. Materials and methods In 42 patients of angiographic intermediate coronary stenosis (diameter stenosis $40 \%-60 \%$ ), IVUS imaging was performed and intracoronary pressure was recorded. The patients were classified as either unstable plaques group $(n=30)$ or stable plaques group ( $\mathrm{n}=12$ ) by IVUS image. The biomechanical properties (distensibility index and stiffness) of coronary artery were calculated and the plaque behaviour during cardiac cycle was determined.

Results There was no significant difference in percent area stenosis between eccentric plaque group and concentric plaque group $(53.9 \pm 8.9 \%$ vs $58.4 \pm 9.8 \%, \mathrm{p}>0.05)$. The coronary distensibility index in unstable plaques was significantly greater than it was in stable plaques $(2.1 \pm 0.3$ vs $1.2 \pm 0.2 \mathrm{~mm} \mathrm{Hg}-1, \mathrm{p}<0.01)$, but stiffness $\beta$ for stable plaques was significantly greater than it was for unstable plaques $(8.1 \pm 1.3$ vs $29.4 \pm 7.2, p<0.01)$. The change of plaque area during cardiac cycle (plaque distensibility) in unstable plaque group was greater than it was in stable plaque group $\left(0.52 \pm 0.22 \mathrm{~mm}^{2}\right.$ vs $\left.0.24 \pm 0.19 \mathrm{~mm}^{2}, \quad \mathrm{p}<0.01\right)$. Positive remodelling occurred more frequently with unstable plaques than with stable plaques (63\% vs $8 \%, \mathrm{p}<0.01$ ).

Conclusion High coronary artery distensibility and high plaque distensibility during the cardiac cycle in eccentric lesions will likely increase plaque vulnerability. 